\title{
Numerical study on the application of vacuum insulation panels and a latent heat storage for refrigerated vehicles with a large Eddy lattice Boltzmann method
}

\author{
Maximilian Gaedtke ${ }^{1,2} \cdot$ Simon Wachter ${ }^{3}$. Sven Kunkel ${ }^{4}$. Sebastian Sonnick ${ }^{4}$ Matthias Rädle ${ }^{4}$. \\ Hermann Nirschl ${ }^{2} \cdot$ Mathias J. Krause ${ }^{1,2,5}$
}

\begin{abstract}
The development of sustainable trucks has drawn a lot of attention lately. However, the reduction of fuel consumption and emissions related to deep frozen food transports has not yet been satisfactorily considered. In this paper, a thermal Large Eddy Lattice Boltzmann Method (LES-LBM) is applied to investigate two concepts for optimized refrigerated vehicles: (a) the inclusion of vacuum insulation panels (VIP) in the refrigerated body's walls and (b) the introduction of a latent heat storage (LHS) to exchange fuel-driven air conditioning (AC), both with conveniently worth while potential to decrease fuel consumption and related emissions. The present numerical method allows for an accurate and efficient transient conjugate heat transfer simulation including the spatial and temporal resolution of the temperature distribution inside the insulation walls and the cargo in addition to the turbulent surrounding air flow induced by the AC. The present concept of VIP inclusion is found capable of halving the required cooling energy. In addition, it effectively reduces the variations in the temperature of the chilled goods during cooling operation, which is an important measure of the quality of the refrigerated body. The reduced required cooling energy is further found to enable the AC system to be replaced by a LHS mounted near the top of the refrigerator body and an additional ventilation system of lower total capacity. A comparison between simulations with conventional AC and LHS is conducted concerning the temperature homogeneity of loaded deep frozen food products. It is shown that a slight flow around the refrigerated goods is necessary and the maximum downtime of the AC system is 8 min in case of combined PUR and VIP insulation and $11 \mathrm{~min}$ in case of an additional LHS.
\end{abstract}

Maximilian Gaedtke

maximilian.gaedtke@kit.edu

1 Lattice Boltzmann Research Group (LBRG), Karlsruhe Institute of Technology (KIT), Karlsruhe, Germany

2 Institute for Mechanical Process Engineering and Mechanics (MVM), Karlsruhe Institute of Technology (KIT), Karlsruhe, Germany

3 Institute for Technical Chemistry (ITC), Karlsruhe Institute of Technology (KIT), Karlsruhe, Germany

4 Center for Mass Spectrometry and Optical Spectroscopy (CeMOS), University of Applied Sciences Mannheim, Mannheim, Germany

5 Institute for Applied and Numerical Mathematics (IANM), Karlsruhe Institute of Technology (KIT), Karlsruhe, Germany

\section{Introduction}

At present, the use of fossil fuels (e.g. coal, oil and gas) is still increasing, due to industrialization of developing nations and growing world population [16]. In the last decades more and more renewable energy systems were developed, using hydro and wind power, geothermal heat, solar energy and others [8]. Supporting the fossil energy revolution towards renewable energy supply, the European Commission (EC) presented a road map up to the year 2050 with suggested actions reducing the carbon dioxide emissions by $80 \%$ to $95 \%$ compared to the level of 1990 [10]. It is argued that to reach these requirements, electricity will play a central role in a future low-carbon economy and that a sole change from fossil fuels to renewable energy resources in the energy production sector is insufficient [29]. 
Recently, the attention on the mobility sector is increased with an initial focus on small passenger cars with combustion engines. As a start hybrid, plug-in hybrid and electric vehicles are developed, enabling the transportation over short distances [4]. Another approach is the production of bio diesel for all heavy weight and transportation vehicles. By using biomass in a gasification process part of the actual diesel consumption can by replaced by bio diesel [7, 8]. The mandatory reduction of the diesel consumption of transportation vehicles may be achieved through an improvement in efficiency of many different small scale processes. One process with conveniently worth while potential to decrease fuel consumption and related emissions is the use of efficiently optimized refrigerated vehicles for the delivery of cooled products. Additional to their fuel-driven locomotion, a fuel-driven cooling unit is used in present vehicles. Furthermore, food delivery to personal homes in times of expanding internet food providers and upcoming ideas for autonomous driving cars will increase permanently and the optimized efficiency of improved insulation systems for refrigerated vehicles is mandatory for energetic as well as economic reasons.

Embedded in a long-term project many different improvement possibilities of the energy consumption for refrigeration are tested. Two promising concepts to reduce the fuel consumption of refrigerated trucks are considered in this paper:

- Concept A - insulation optimization using vacuum insulation panels (VIP),

- Concept B - passivation of the cooling effect by latent heat storages (LHS).

Concept A replaces previous used polyurethane rigid foam insulation at all possible locations through vacuum isolation panels (VIP). These panels are characterized by much lower heat conduction and at the same time lower thickness. VIP are filled and mechanically stabilized by highly porous grid structures made of polyurethane or silicates which are on both sides covered by a stable housing and evacuated. Through the porous grid, heat transfer through the panel can be reduced to values as low as the heat conduction of the branched structure plus radiation [27]. Thus, thermal conductivities five to ten times lower than for traditional insulation are achieved [34].

VIP were firstly applied in the construction industry for thermal insulation of external facades. Here filling materials could be used without high static load capacity and danger of vibrations. Initial test methods were applied to describe the average thermal conductivity of VIP. With the help of these test measurements, local differences in thermal conductivity were detected. This is known as the edge effect, whereby a high proportion of the heat conduction takes place through the frame of the panels [32]. Since the first experimental tests, most diverse studies on the thermal conductivity of the panels have been carried out with models for calculating the heat transfer of different panel types [25]. In addition to the core material, a dependence on the temperature level, internal pressure, gas type, permeability and water content in the internal residual air were found to be possible influencing parameters of thermal conductivity $[6,23]$. Since the publication of the first reviews on VIPs, new areas of application have been identified, most notably the cooling and hot water technology [19]. In 2012, Bouquerel et al. [6] proposed a model and applied it to describe combined heat transfer phenomena for determining an average thermal conductivity. They identified the main modeling disadvantages to be the assumptions of a regular structure and the Fourier law. Addressing these drawbacks, Ross-Jones et al. [24] presented a pore scale model based on resolved packed bed simulations with a Lattice Boltzmann Method (LBM) taking into account solid and gas phase heat conduction as well as thermal radiation.

In concept B the fuel-driven cooling unit is exchanged with a LHS filled with a phase change material (PCM). Extensive reviews on the modeling and simulation of thermal behavior as well as initial tests on combinations of VIP and LHS have been carried out by Ahmad et al. [3, 31]. Zalba et al. [35] showed a compilation of various phase change materials including solutions of salts with a suitable temperature range for the transport of refrigerated goods.

Due to the fact that prototypes of refrigerated bodies with built-in AC are expensive in material and manufacturing costs, computational fluid dynamics are used to investigate both concepts before actually constructing a prototype. In addition, spatially resolved measurements without influencing the wall properties or the flow properties in the system are costly if not hardly possible. In a previous study Gaedtke et al. [11] showed the applicability of a double distribution thermal LBM to the simulation of a refrigerated vehicle's insulation. By replacing the widely applied Reynolds averaging turbulence model with a large eddy turbulence model and transiently resolving the flow field, the refrigerated vehicle's walls and their conjugate heat transfer, they found much closer accordance to measured data than previous studies $[17,26]$. The application of the inherently transient LBM to large eddy simulations (LES) has shown to provide a significant speedup and parallel scalability over traditional Finite Volume Methods, see for example in the work of Barad et al. [5], where a speedup of $\sim 12$ is achieved by LBM over FVM for simulations on similar grids and with similar accuracy. With this scalable and fast solver and successful validation of realistic velocity and temperature distributions in the interior of a refrigerated body it is consistent to employ the same simulation method in this study. 
The structure of this paper is following the subsequent line of reasoning: Section 2 introduces the applied thermal LBM including the sub-grid scale model (Section 2.4) and used boundary conditions (Section 2.5). The refrigerated body's geometry, material properties and initial conditions are presented in Section 3, where three different simulation case setups are introduced: conventional PUR insulation (Section 3.1), improved PUR+VIP insulation (Section 3.2) and additional inclusion of a LHS (Section 3.3). In Section 4 the results of the two introduced concepts are discussed by comparison to the conventional PUR insulation case. Mass and heat flux simulations of the two insulation concepts are presented and an application on the leakage of rear doors is shown. Afterwards, the passivation of the temperature field is numerically assessed by an exchange of the cooling unit through the LHS. For this purpose six trolleys with deep-frozen goods, which are modeled by the material values of pure ice, are included in the simulation. The cargo temperature's sensitivity considering switching off the cooling engine is compared for three different insulation and cooling options by flow and temperature fields within the loaded refrigerated vehicle.

\section{Mathematical modeling}

In this work we use the double distribution thermal LBM (DDT-LBM) including a Smagorinsky turbulence model as described in detail in our previous study [11], in which we also presented a rigorous validation of the model itself as well as its implementation in the open source code OpenLB. This validation study included

- a grid convergence study showing that the scheme is second order in space by comparing it with the analytical solution of the porous plate problem,

- a comparison with the benchmark of natural convection in a square cavity for different Rayleigh numbers from laminar to turbulent natural convection,

- and a comparison with measurements for the refrigerated transporter, which is also investigated in the current manuscript.

All three tests showed good agreement with the respective reference values. The method used is briefly introduced in the following Sections 2.1 to 2.4 , while the reader is referred to the original article for a detailed description and validation of the method.

Two distribution functions $f_{i}$ and $g_{i}$ are introduced in Sections 2.1 and 2.2 to solve the mass, momentum and energy equation on the macroscopic scale. The Smagorinsky sub-grid scale model is described in Section 2.4. It adds an eddy viscosity and an eddy diffusivity, which both are incorporated into the Lattice Boltzmann equation (LBE) by modifying the relaxation times $\tau_{f}$ and $\tau_{g}$.

Note that the LBEs presented in the following are solved in their non-dimensional form. Thus, all physical quantities are converted to so called lattice units before the simulation and converted back before post processing. Krüger et al. provide an overview on the dimensionalization of the LBE including complete instructions on how to choose corresponding conversion factors in Chapter 7 in [20].

\subsection{LBM for mass and momentum equation}

The Single Relaxation Time Lattice Boltzmann Equation (SRT-LBE) including a force term $F_{i}(x, t)$ is given by

$f_{i}\left(x+e_{i} \Delta t, t+\Delta t\right)-f_{i}(x, t)=\frac{\Delta t}{\tau_{f}}\left(f_{i}(x, t)-f_{i}^{e q}(x, t)\right)+F_{i}(x, t)$

with the discrete probability function $f_{i}$, the space and time coordinates $x$ and $t$, the discrete velocity $e_{i}$, direction index $i$, and the equilibrium distribution function

$f_{i}^{e q}(\rho, u)=w_{i} \rho\left(1+\frac{u \cdot e_{i}}{c_{s}^{2}}+\frac{\left(u \cdot e_{i}\right)^{2}}{2 c_{s}^{4}}-\frac{u \cdot u}{2 c_{s}^{2}}\right)$.

Herein the weights $w_{i}$, the discrete velocities $e_{i}$ and the speed of sound $c_{s}$ are given by the standard D3Q19 velocity set [20]. The relaxation time $\tau_{f}$ in Eq. 1 is given as a function of the kinematic shear viscosity $v$ with

$\tau_{f}=\frac{v}{c_{s}^{2}}+\frac{1}{2}$.

The macroscopic values of density $\rho$ and velocity $u$ are calculated from the distribution function's moments

$\rho(x, t)=\sum_{i=0}^{18} f_{i}(x, t) \quad$ and $\quad u(x, t)=\frac{1}{\rho} \sum_{i=0}^{18} e_{i} f_{i}(x, t)$.

\subsection{LBM for energy equation}

With the similarities between the advection-diffusion equation (ADE) and the incompressible Navier-Stokes equations, it is evident to use an analogous Lattice Boltzmann algorithm for advection-diffusion problems: The SRT-LBE for the energy equation is given by

$g_{i}\left(x+e_{i} \Delta t, t+\Delta t\right)=g_{i}(x, t)-\frac{\Delta t}{\tau_{g}}\left(g_{i}(x, t)-g_{i}^{\mathrm{eq}}(x, t)\right)$ 
with the discrete temperature distribution function $g_{i}$ in discrete direction $i$, the equilibrium distribution function

$g_{i}^{\mathrm{eq}}(T, u)=w_{i} T\left(1+\frac{e_{i} \cdot u}{c_{s}^{2}}\right)$

and the macroscopic temperature $T$. The velocity $u$ is provided by Eq. 4.

While the LBM for the NSE requires preserving up to first order moments (mass and momentum, see Eq. 4), the LBM for the ADE does only need to preserve the zeroth order moment (temperature, see Eq. 6). The first order moment of the LBM for the ADE calculates to $T u$, which is not conserved during the collision operation stated above given that $u$ is calculated from the distribution $f$ rather than from $g$. Thus, using the D3Q7 lattice set is sufficient [2].

The relaxation time $\tau_{g}$ is given as a function of the diffusion coefficient $\kappa$ by

$\tau_{g}=\frac{\kappa}{c_{s}^{2}}+\frac{1}{2}$

similar to the viscosity in the NSE (for example see Krüger et al. [20]).

The macroscopic temperature and heat flux $Q$ are given by

$T(x, t)=\sum_{i=0}^{6} g_{i}(x, t) \quad$ and $\quad Q(x, t)=-A \lambda \nabla T(x, t)$.

Herein, $A$ is the surface area over which the heat flux is calculated and $\lambda$ is the thermal conductivity.

\subsection{Coupling by Boussinesq approximation}

In order to model the interactions between the velocity and temperature fields a coupling procedure is introduced. Guo et al. present a coupled LBM with the BGK collision operator in [12] based on the Boussinesq approximation. Therein, the force term according to Eq. 1 is set to be the Boussinesq buoyancy force as

$F(x, t)=-\rho_{0} \beta g\left(T(x, t)-T_{0}\right)$,

where $\rho_{0}$ and $T_{0}$ are the reference density and temperature, respectively, and the lattice forcing term $F_{i}$ is calculated as a function of $F$ with the forcing scheme presented by Guo et al. [13].

\subsection{Large Eddy turbulence model}

As in our previous work [11], the Smagorinsky sub-gridscale model introduced to the LBM by Hou et al. [15] is used. Following Hou et al., the viscosity $v$ in Eq. 3 is replaced by the effective viscosity $\nu_{\text {eff }}$, which is defined as the sum of molecular viscosity $v_{0}$ and eddy viscosity $v_{t}$ with

$v_{\text {eff }}=v_{0}+v_{t}=v_{0}+\left(C_{S} \Delta\right)^{2} \sqrt{2 \sum_{\alpha, \beta} S_{\alpha \beta} S_{\alpha \beta}}$,

with the Smagorinsky constant $C_{S}=0.1$ and the filter width $\Delta=\Delta x$. The strain rate $S_{\alpha \beta}$ is computed locally using the second moment of the non-equilibrium distributions with

$S_{\alpha \beta}=-\frac{1}{2 \rho \tau_{f, \mathrm{eff}} c_{s}^{2}} \sum_{i=0}^{18} e_{i, \alpha} e_{i, \beta}\left(f_{i}-f_{i}^{e q}\right)$

and $\tau_{f \text {,eff }}=\frac{v_{\text {eff }}}{c_{s}^{2}}+\frac{1}{2}$ denoting the modified relaxation time as a function of $v_{\text {eff }}$.

The thermal diffusivity $\kappa$ in Eq. 7 is analogously replaced by the effective thermal diffusivity given by

$\kappa_{\mathrm{eff}}=\kappa_{0}+\kappa_{t}=\kappa_{0}+\frac{\nu_{t}}{\operatorname{Pr}_{t}}$

where the turbulent thermal diffusivity $\kappa_{t}$ is calculated by the turbulent Prandtl number

$\operatorname{Pr}_{t}=\frac{v_{t}}{\kappa_{t}}=0.86$

\subsection{Boundary conditions}

As in our previous work [11] Latt's [22] regularized boundary approach is used for pressure and velocity boundary conditions. A constant temperature boundary condition is implemented as described in [20] and the outflow boundary condition for the temperature is used as recommended by Junk and Yang [18]. Simple bounce back [28] is used for no-slip and adiabatic boundaries. The half lattice division scheme described by Wang [33] is utilized to simulate conjugate heat transfer, where the diffusion equation and the advection-diffusion-equation are solved in solid and fluid regions, respectively, on a single mesh.

\section{Geometry, material properties and simulation case setups}

\subsection{Refrigerated body geometry and conventional PUR insulation}

A refrigerated body for the commercial vehicle MercedesBenz Sprinter 316 is applied in this study. The refrigerated body itself is the model CoolerBox 2.0 TK $3500 \mathrm{HS}$ from Kress Fahrzeugbau $\mathrm{GmbH}$ with a double wing rear door and polyurethane hard foam (PUR) insulation walls as shown in the schematic representation in Fig. 1. The respective wall thicknesses and dimensions of the cooling unit are summarized in Table 1. The Carrier Xarios 500T cooling 
Fig. 1 Schematic representation of the refrigerated body and the built-in AC system. The AC's outlet and suction side are shown in green and orange, respectively. Adapted from [11]

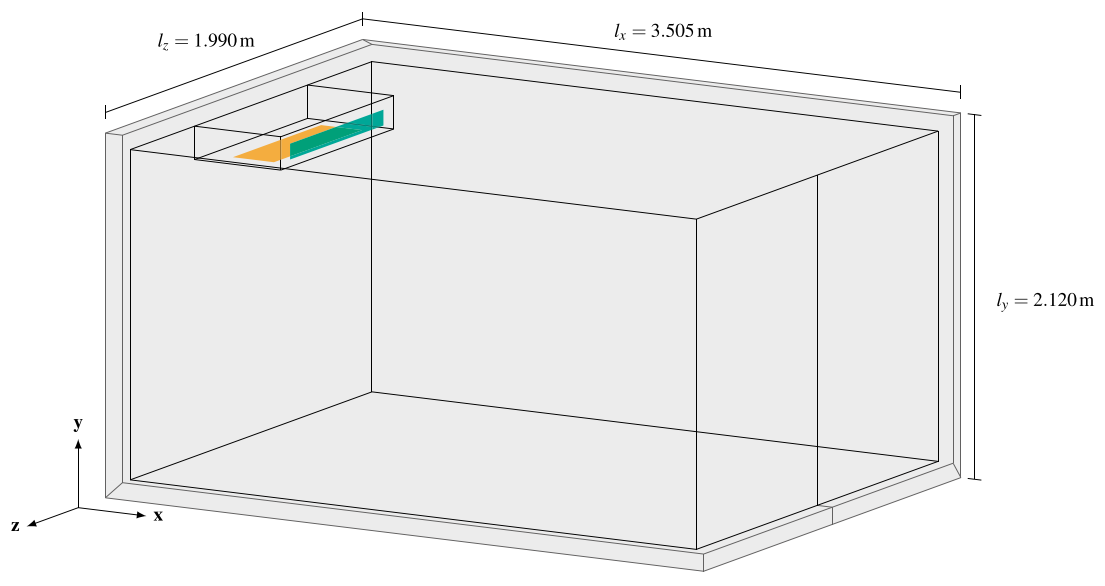

unit is installed, which can be used for both, heating and cooling purposes with a full-load volume flow of $\dot{V}=$ $990 \mathrm{~m} / \mathrm{h}^{3}$ and a heating and cooling capacity of $Q=$ $950 \mathrm{~W}$. The energy supply of the transport refrigeration system can be provided either by the diesel engine installed in the vehicle during the journey or externally by $400 \mathrm{~V}$ three-phase current during longer rest periods.

The material properties of the internal air and the PUR insulation are given in Table 2. At $t=0$ the velocity in the simulation domain is set to $0 \mathrm{~m} / \mathrm{s}$, while the internal air temperature is set to $-20{ }^{\circ} \mathrm{C}$. The insulation walls are set to $5{ }^{\circ} \mathrm{C}$ and the outer surface of the refrigerated body is assumed to have a constant temperature of $30^{\circ} \mathrm{C}$. It should be mentioned, that the latter represents a worst case scenario for the central European food transport.

The AC flow rate and cooling capacity are adjusted according to the manufacturer information by calculating appropriate velocity and temperature values for the $\mathrm{AC}$ outlet boundary every 50 time steps. In order to ensure a stable simulation, the AC flow rate is ramped up to its desired value over a time span of $20 \mathrm{~s}$.

The challenge achieving a physical simulation time of 60 $\mathrm{s}$ and more is passed using massive parallel execution of the LB solver on a cluster computer, namely the KIT research high-performance computer ForHLR II. For the scope of this study, the refrigerated body is discretized with about 65 million grid points, which requires eight hours calculation time on 1000 allocated CPU cores for a physical simulation time of $60 \mathrm{~s}$. With the chosen lattice Mach number of
$\mathrm{Ma}=0.2$ a physical simulation time of $60 \mathrm{~s}$ corresponds to about half a million time steps.

The geometry and model described above have been validated successfully against transient experimental data for a period of $60 \mathrm{~s}$ from 17 characteristic measuring points in the refrigerated body, 13 for the temperature distribution and 4 for the velocity distribution with absolute errors below $0.4 \mathrm{~m} / \mathrm{s}$ and below $1 \mathrm{~K}$, respectively. The execution of the validation study and its results have been described in detail in our previous work [11].

The conventional PUR insulation is considered as a reference case; its simulation setup is referred to as $P U R$ in the following.

\subsection{Integration of vacuum insulation panels}

For the largest possible integration of the VIPs without changing the outer design of the refrigerated body, the new insulation is mounted in all parts of walls, where no interfering elements such as hinges, edges, corners and brackets exist. A schematic illustration of the resulting insulation concept applying VIPs is shown in Fig. 2. Herein, light gray elements show a framework made from hard foam insulation, while clear areas are filled with VIPs in the following simulations.

This insulation concept cannot be realized with conventional VIP elements. To ensure an elegant integration into the refrigerated box, the silica-based core material mixture is pressed directly into a frame made of PUR. The walls are
Table 1 Summary of the geometric dimensions of the refrigerated body, the $\mathrm{AC}$, its outlet $(\mathrm{O})$ and suction side $(\mathrm{S})$

\begin{tabular}{|c|c|c|c|c|c|c|c|}
\hline \multicolumn{4}{|c|}{ Refrigerated body } & \multicolumn{4}{|c|}{ Air conditioning unit } \\
\hline \multicolumn{2}{|l|}{ Dimensions } & \multicolumn{2}{|l|}{ Wall thicknesses } & \multicolumn{2}{|l|}{ Dimensions } & \multicolumn{2}{|l|}{ Openings } \\
\hline Length: & $3.505 \mathrm{~m}$ & Ground: & $0.1 \mathrm{~m}$ & Length: & $0.505 \mathrm{~m}$ & (S) Length: & $0.12 \mathrm{~m}$ \\
\hline Height: & $2.120 \mathrm{~m}$ & Ceiling: & $0.1 \mathrm{~m}$ & Height: & $0.194 \mathrm{~m}$ & (S) Width: & $0.79 \mathrm{~m}$ \\
\hline Width: & $1.990 \mathrm{~m}$ & Front: & $0.1 \mathrm{~m}$ & Width: & $0.874 \mathrm{~m}$ & (O) Height: & $0.06 \mathrm{~m}$ \\
\hline & & Rear Door: & $0.085 \mathrm{~m}$ & & & (O) width: & $0.79 \mathrm{~m}$ \\
\hline & & Sides: & $0.065 \mathrm{~m}$ & & & & \\
\hline
\end{tabular}


Table 2 Material properties (thermal conductivity $\lambda$, density $\rho$, specific heat capacity $c_{p}$, thermal expansion coefficient $\beta$ and kinematic viscosity $\nu$ ) for air according to VDI Heat Atlas [30] and for the insulation materials PUR and VIP according to $[9,21,30]$

\begin{tabular}{llllll}
\hline & $\lambda$ & $\rho$ & $c_{p}$ & $\beta$ & $v$ \\
& $10^{-3} \mathrm{~W} /(\mathrm{m} \mathrm{K})$ & $\mathrm{kg} / \mathrm{m}^{3}$ & $10^{3} \mathrm{~J} /(\mathrm{kg} \mathrm{K})$ & $10^{-3} 1 / \mathrm{K}$ & $10^{-6} \mathrm{~m}^{2} / \mathrm{s}$ \\
\hline Air & 22.62 & 1.378 & 1.007 & 3.974 & 11.766 \\
PUR & 23.00 & 39.50 & 1.400 & - & - \\
VIP & 7.00 & 1140 & 0.746 & - & - \\
\hline
\end{tabular}

made of glass fibre reinforced plastic (GRP), comparable to conventional refrigerated boxes, to ensure the mechanical stability required for this application.

The aforementioned edge effect observed with conventional VIPs is mainly caused by the foil packaging containing metallized layers to reduce gas diffusion through the envelope. This effect is negligible in the present concept as no metallised foil is used. In order to compensate for an inevitable increase in internal pressure over time, it is possible to re-evacuate the VIP elements at a later date using an integrated pump.

The surface area replaced by VIPs is calculated to $21.672 \mathrm{~m}^{2}$ replacing $58.2 \%$ of the body's outer or $67.2 \%$ of it's inner surface. The material properties of VIP insulation used are given in Table 2.

This simulation setup including VIP insulation is referred to as $P U R+V I P$ in the following.

\subsection{Integration of a latent heat storage}

The LHS is $2.00 \mathrm{~m} \times 1.40 \mathrm{~m} \times 0.06 \mathrm{~m}(1 \times \mathrm{w} \times \mathrm{h})$ in size and filled with $95.4 \mathrm{~kg}$ of PCM made from an eutectic mixture of water and sodium chloride with a salt mass fraction of $23.3 \%$, a melting temperature of $-21^{\circ} \mathrm{C}$ and latent heat of

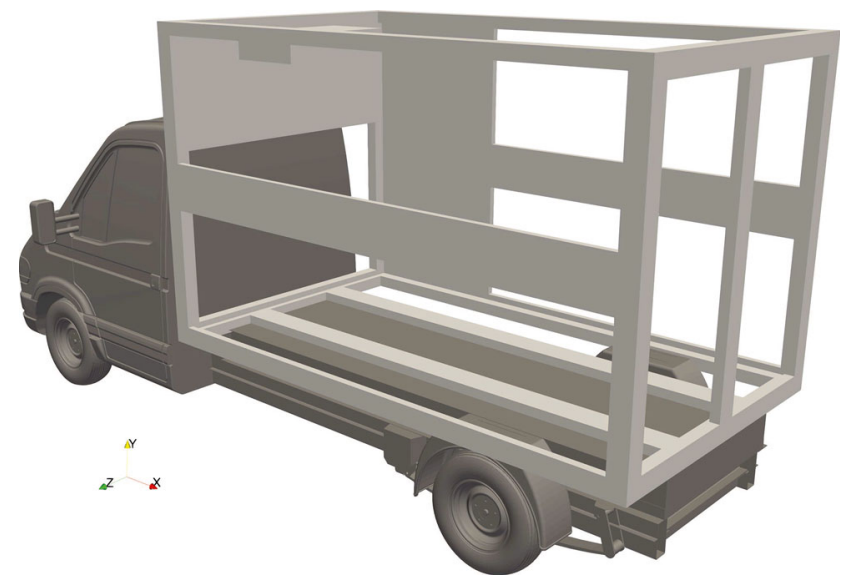

Fig. 2 VIP integration concept: Schematic illustration of the refrigerated body. Light gray areas represent the framework made of conventional PUR insulation, the clear areas are filled with VIPs
$233 \mathrm{~kJ} / \mathrm{kg}$ [14]. With a total cooling power of $6.175 \mathrm{kWh}$, its size and mass are designed to provide the required refrigeration capacity for an eight hour long-distance transport following the DIN 8959 [1] including a 75\% ATP safety factor. The LHS is not permanently integrated into the cooling structure, but is designed using individual modules and can therefore be used and exchanged very flexibly. Thus, in practice, either pre-cooling of the PCM in an external cooling chamber or crystallization within the truck with a cooling unit is possible.

This enables a straightforward implementation of the LHS into the simulation by assuming the PCM to constantly have the melting temperature. The LHS is mounted in the refrigerated body's interior, $0.05 \mathrm{~m}$ under the ceiling and centered in $x$ and $z$ direction.

This simulation setup including VIP insulation and the LHS is referred to as $P U R+V I P+L H S$ in the following.

\section{Results and discussion}

In this section, the results of the proposed concepts and applications are presented. Section 4.1 shows the investigations on an improvement of the wall insulation through a partly exchange with VIPs. Additionally, an application on the leakage of a cooler box door is shown and discussed. In Section 4.2 the exchange of the AC system is introduced, as well as the influence of this replacement on the flow and temperature fields in a loaded vehicle. Eventually, the sensitivity of the loading temperature for differently isolated refrigerated bodies is discussed, taking into account switching off the AC.

\subsection{Concecpt A - insulation optimization using vacuum insulation panels (VIP)}

\subsubsection{Comparison of the insulation effect}

The ingoing heat flux through the walls of the cooled box is an important measurement in order to compare different insulation materials applied in a refrigerated vehicle's wall. It is possible to calculate the spatial heat transfer in normal direction through the walls for every grid point by resolving the insulation walls as a structure of specific material. In order to compare the heat fluxes, the resolved grid with PUR and PUR+VIP insulation is given in Fig. 3. The flow fields from the simulations with and without VIP show a nearly identical velocity distribution as in [11, Figure 11], which is why no illustration is given here.

Figure 3 (left) shows high differences between the edges of the refrigerated body with $Q=0 \mathrm{~W}$ to $4 \mathrm{~W}$ and the surfaces with $Q=10 \mathrm{~W}$ to $13.6 \mathrm{~W}$. The heat flow through the insulation walls is shown in the simulation. Furthermore, 
Fig. 3 Heat flux in wall normaldirection through the PUR insulation (left) and through the PUR+VIP insulation (right)
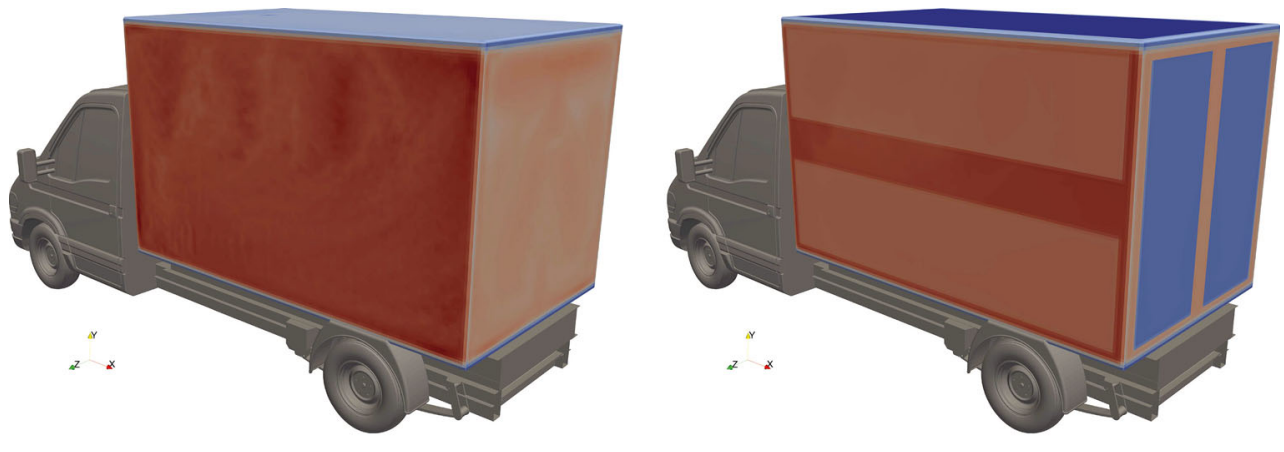

the differences in the wall thicknesses are recognizable, since the side walls with their smaller thickness, in contrast to the ceiling and front, allow for a higher heat flow.

Figure 3 (right) shows the heat flow chart for simulation case with VIPs. The reduced ingoing heat flow of the area of the VIPs is clearly visible, which has dropped from $Q=10 \mathrm{~W}$ to $13.6 \mathrm{~W}$ to $Q=0 \mathrm{~W}$ to $5 \mathrm{~W}$. In addition, it becomes apparent that with the help of the new insulation concept, hardly any heat penetrates into the refrigerator car via the ceiling, even at the edges to other walls, whereas the heat flow via the rigid foam insulation of the side wall has maximum values of up to $Q=13.6 \mathrm{~W}$.

With the help of Nusselt correlations from the VDI Heat Atlas [30], a rough expectation for the total heat flux was calculated. For this purpose, a correlation for the overflow of planar surfaces is used for interior space on all internal walls separately and averagely as seen in [11]. For each wall, a specific mean Reynolds number is calculated, using the velocities from the validation case in [11]. For the PUR insulation a total incoming heat flow of $Q=470 \mathrm{~W}$ and for PUR+VIP an incoming heat influx through the wall of $Q=$ $274 \mathrm{~W}$ is determined by the correlations. The simulated heat flux through the wall sums up to $Q=458 \mathrm{~W}$ for the PUR and $Q=248 \mathrm{~W}$ for the PUR+VIP insulation, respectively. Thus, the relative deviations from the Nusselt correlation is around $2.6 \%$ and $9.5 \%$, respectively.

The variation of the insulation material changes the incoming heat flux in the proposed set-up of more than $54.15 \%$. This leads to a significant reduction in the cooling energy consumed, which considerably improves the operating costs of the truck and facilitates the provision of the complete cooling energy for a one-day delivery cycle through a LHS system. This is investigated numerically in Section 4.2.1.

\subsubsection{Influence of leakage in the rear door}

One of the possible leakage positions in a refrigeration structure is the tailgate insulation due to its mechanical

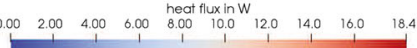

stress by opening and closing. Leakage in technical systems describes the undesired entry or exit of solids, liquids or gases [24]. In the event of the leakage of a refrigerated transporter, cooled indoor air could leave the isolated space and warmer outside air could penetrate into the structure. This would cause a loss of cooling energy depending on the size of leakage a high degree of cooling performance of the AC to the environment. With the chilled airflow impinging at high speed at the top of the door, additional performance losses are expected in comparison with leaks in areas of lower velocity. In order to analyze a door leakage an exemplary outflow opening of $l_{z \text {, leak }}=0.0125 \mathrm{~m}$ width and $l_{y \text {, leak }}=1.92 \mathrm{~m}$ height is provided at the closing seam of the tailgate at position $z=\frac{l_{z}}{2}$. This corresponds to a total leakage area of $A_{\text {leak }}=0.024 \mathrm{~m}^{2}$.

The resulting velocity field in the leakage area is shown in Fig. 4. As expected, a significantly higher outflow velocity in the upper part of the door than in the remaining opening is predicted by the simulation. In comparison, however, in a section of the refrigerated body at position $z=\frac{l_{z}}{2}$ no increased outflow velocity in the $x$-direction can be determined. The heat flow entering the refrigerated body is determined by means of a time averaged volumetric flow within the simulation. In order to avoid a measurement of the inlet state, the volumetric flow measurement starts after a physical time of one minute and the calculated heat flow is averaged over a time of one minute, while ensembles are recorded every five seconds.

The simulation results in an average heat flow of $Q_{\text {leak }}=545 \pm 29 \mathrm{~W}$ entering the cooling transporter via the modeled leakage. The fluctuations are caused by the different volume flow rates, which are themselves caused by the turbulence of the flow. Within the measuring time of one minute maximum and minimum incoming heat flow are $Q_{\text {leak,max }}=606.5 \mathrm{~W}$ and $Q_{\text {leak,min }}=487.6 \mathrm{~W}$.

A leakage of this size represents a considerable contribution to the loss of power and exceeds the loss of cooling capacity via the simulated rigid foam insulation. Taken together with the simultaneous heat loss via the 

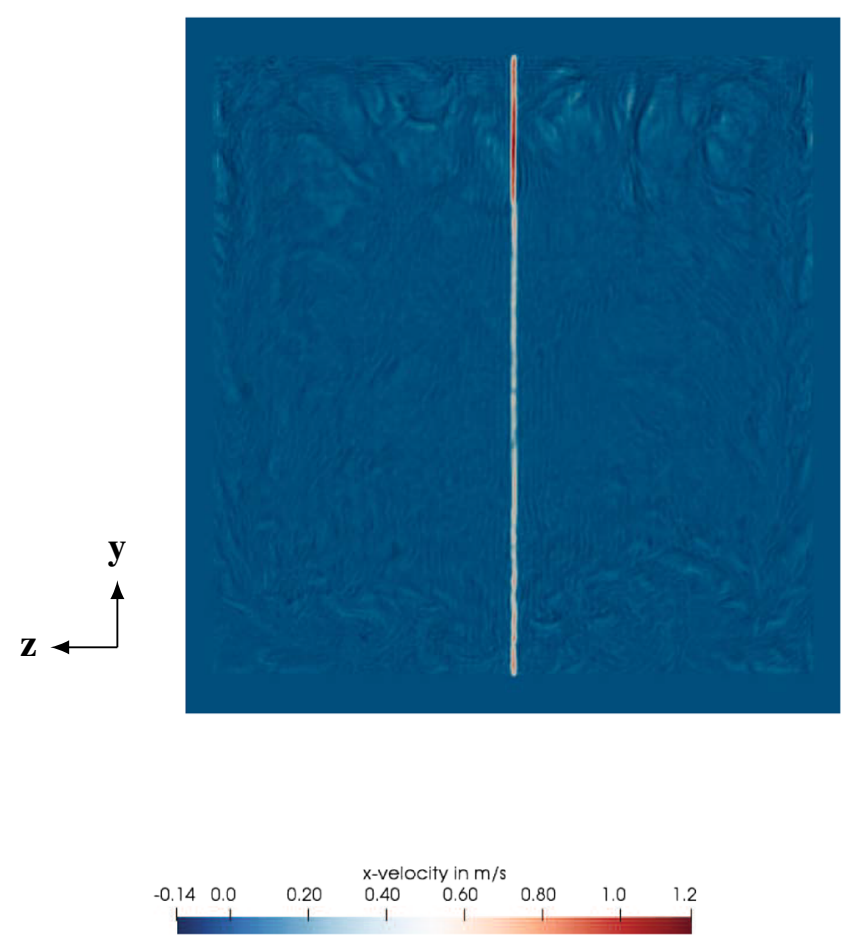

Fig. 4 Distribution of the velocity's $\mathrm{x}$ component near the rear wall with leakage through rear door, slice in the y-z-plane rigid foam insulation, theoretically more than the cooling capacity of the air-conditioning system would be lost to the environment, which implies continuous operation of the air-conditioning system under full load. The interior of the refrigerated body would heat up over a longer period of time, as the heat flow cannot be compensated completly. This would lead to considerable fuel costs, high carbon dioxide emissions and eventually spoilage of the goods and must be avoided urgently in practice.

\subsection{Concecpt B - passivation of the cooling effect by latent heat storage (LHS)}

\subsubsection{Influence of passive cooling on flow field and loading temperatures}

Given that the maximum temperature of the chilled goods after $60 \mathrm{~s} \mathrm{cooling} \mathrm{under} \mathrm{full} \mathrm{load} \mathrm{is} \mathrm{a} \mathrm{useful} \mathrm{measure} \mathrm{of} \mathrm{the}$ quality for the cooling performance, it is worth to analyze this criterion numerically and apply it to the presented concepts. For this purpose six grid trolleys with deepfrozen goods are included in the simulation. The assumed refrigerated cargo is deep-frozen food, which is modeled by the material values of pure ice given in [30] with a thermal
Fig. 5 Streamline representation of the simulated air flow inside the refrigerated vehicle including surfaces temperatures of the loading with PUR insulation

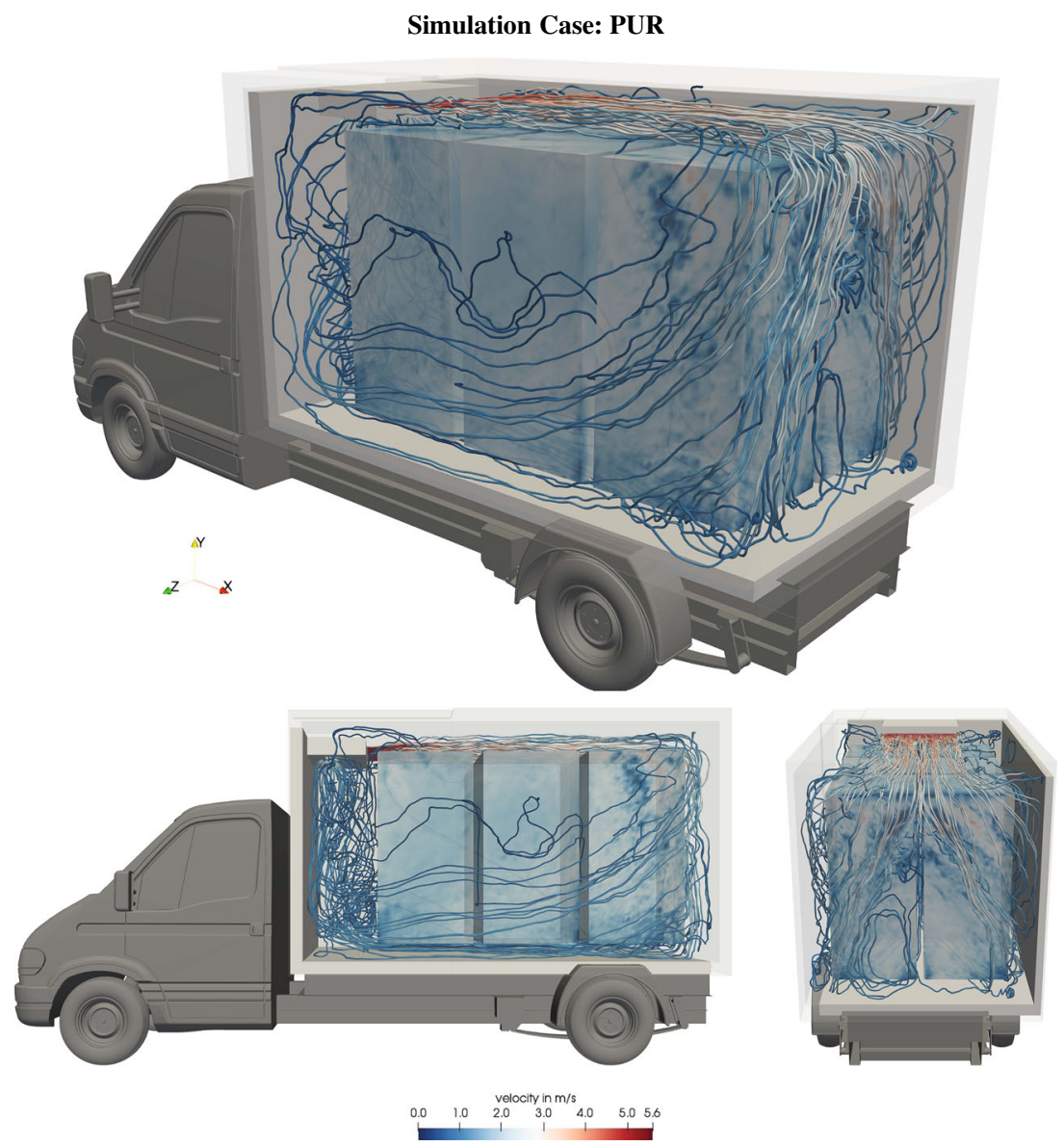


conductivity of $\lambda=512.6 \times 10^{-} \mathrm{W} /(\mathrm{m} \mathrm{K})$, a density of $\rho=993.6 \mathrm{~kg} / \mathrm{m}^{3}$ and a specific heat capacity of $c_{p}=4401 \mathrm{~J}(\mathrm{~kg} / \mathrm{K})$. The goods are stacked virtually on a grid trolley, which dimensions are given with $0.72 \mathrm{~m} \times$ $0.81 \mathrm{~m} \times 1.62 \mathrm{~m}(\mathrm{l} \times \mathrm{w} \times \mathrm{h})$. In addition, a gap width of $0.05 \mathrm{~m}$ between the trolleys is kept for load securing and a gap width of $0.12 \mathrm{~m}$ is kept at the bottom due to the rolls (not resolved here). Since the underside of the AC must be kept clear of cargo in order to properly suck in indoor air, a distance of $0.60 \mathrm{~m}$ is recommended between the inside front wall and the closest trolley.

Figure 5 shows the flow of the internal air around these chilled cargo by means of streamlines and with regular PUR insulation, where significant differences to the flow of the empty refrigerated body become visible, compare in particular Figure 11 in [11]. The flow field in Fig. 5 is comparable to that of an empty refrigerated body up to just below the body's rear edge. The average velocity drops from $2.90 \mathrm{~m} / \mathrm{s}$ to less than $1.45 \mathrm{~m} / \mathrm{s}$. There is also an all-round flow both under the trolleys and between the side walls and the trolleys. The diversion of part of the flow into the central channel between the trolleys can also be observed. Comparatively low air movement can be detected for the air in the vertical spaces between the cargoes, while the formation of temperature inhomogeneities in these spaces is mostly avoided in spite of high flow rate around the cargo.

Figure 6 shows streamlines of the convection generated by the air-conditioning system with VIP. When compared against the case of PUR insulation shown in Fig. 5, a more uniform and cooler surface temperature of the chilled goods is achieved in the VIP insulation case. This clearly shows an advantage of the new insulation concept. The case with LHS was simulated with a quarter of the volume flow of the AC system and without active cooling. This case is shown in Fig. 7. In comparison with Fig. 6, a good flow around the refrigerated goods is ensured even with only a quarter AC power.

The cargo's internal temperature distributions are shown in Fig. 8 for all three cases by means of isotemperature contours. With a minimum and maximum temperature of $253.15 \mathrm{~K}$ to $254.65 \mathrm{~K}$, the PUR insulation results in the largest temperature variation of $1.5 \mathrm{~K}$, while the PUR+VIP and the LHS cases show temperature variations of $0.26 \mathrm{~K}$ and $0.66 \mathrm{~K}$. Figure 8 (left) illustrates much larger inhomogeneities of the cargo's temperature distribution with PUR insulation compared to the two VIP insulation
Fig. 6 Streamline representation of the simulated air flow inside the refrigerated vehicle including surfaces temperatures of the loading for the simulation case PUR+VIP

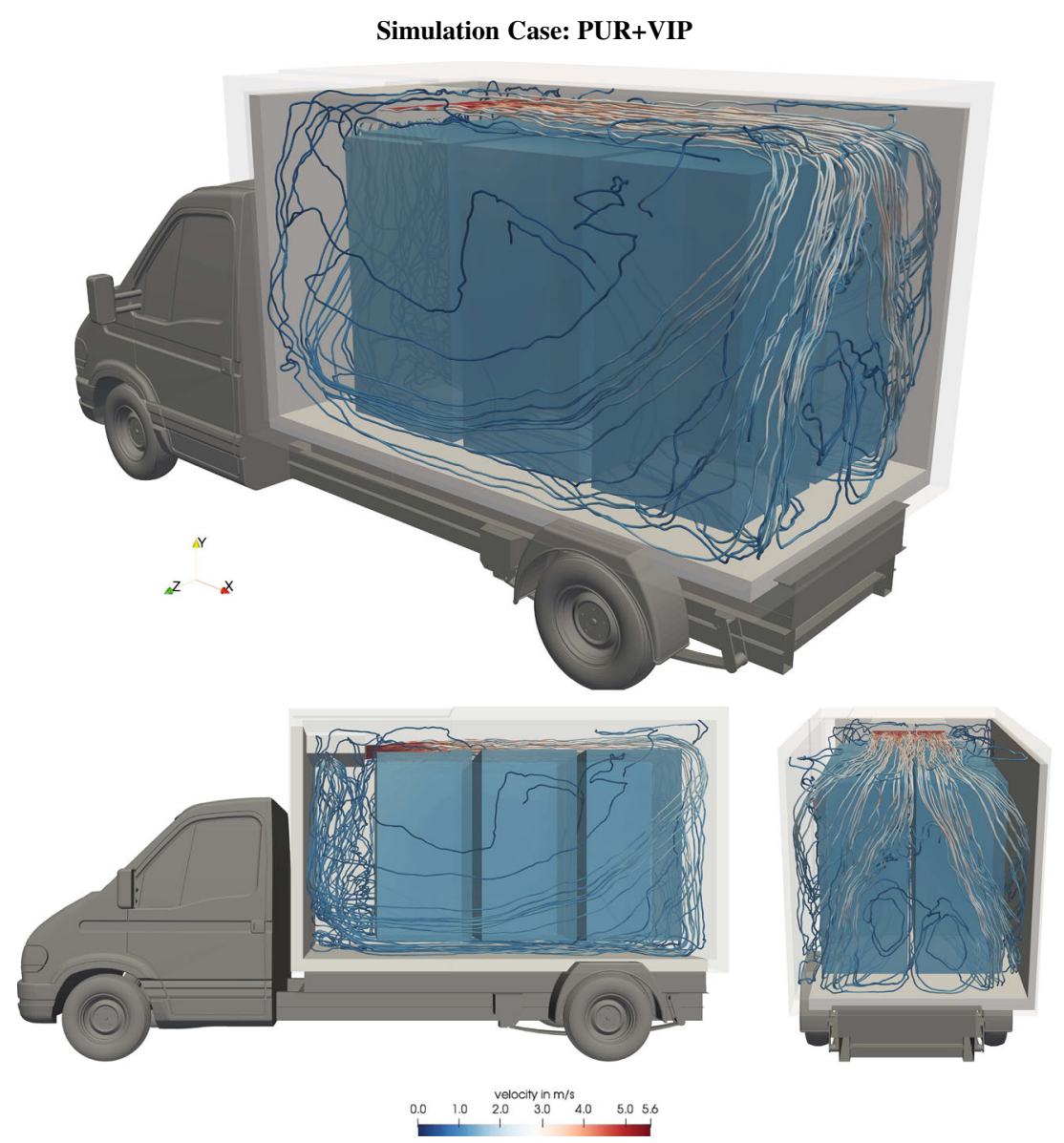


Fig. 7 Streamline representation of the simulated air flow inside the refrigerated vehicle including surfaces temperatures of the loading for the simulation case PUR+VIP+LHS

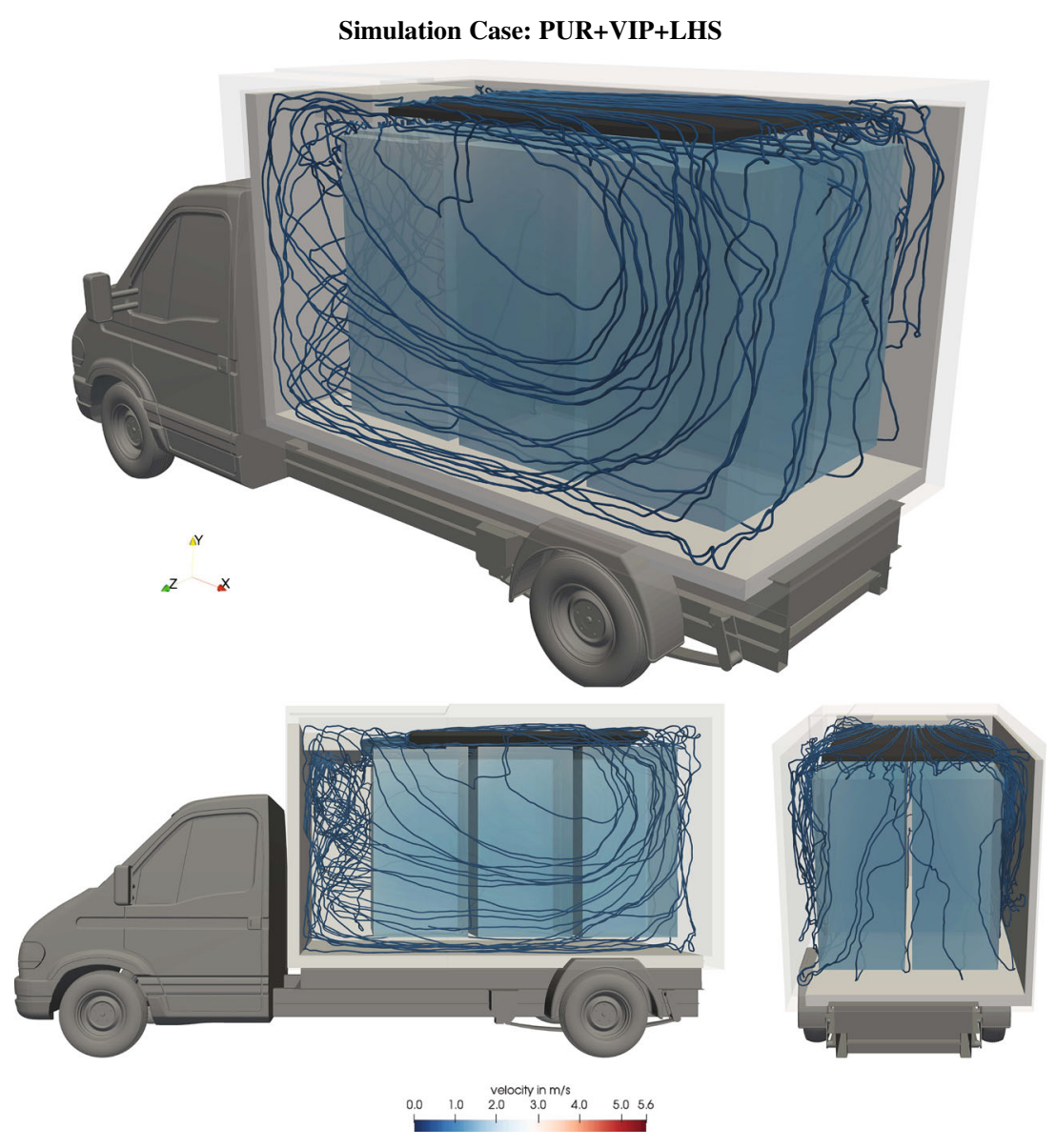

cases, while the reduced AC flow of the LHS case (Fig. 8 right) results in slightly higher inhomogeneity than in the PUR+VIP case. Particularly in the case of PUR+VIP insulation (Fig. $8 \mathrm{mid}$ ), the locally different cooling effect can be detected, where the coolest temperature follows the surrounding flow (compare Fig. 6 bottom right). This is also visible for the LHS case, which directly recommends the use of an active flow. However, while a relatively narrow outlet of the AC system is used here, ventilation across the entire width of the refrigerated body seems to make sense for a more even flow around the LHS and thus also around the chilled goods. The surface temperature of the chilled goods
PUR

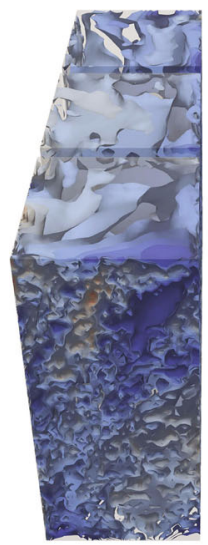

PUR+VIP

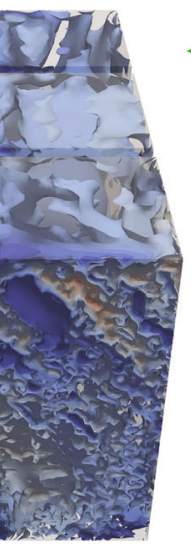

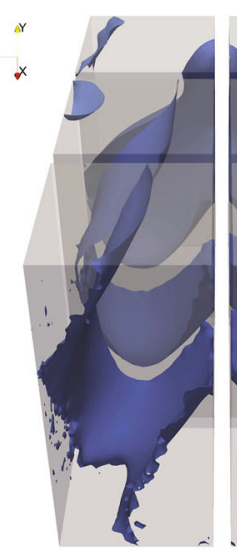

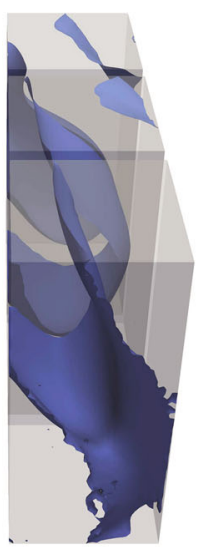

PUR+VIP+LHS

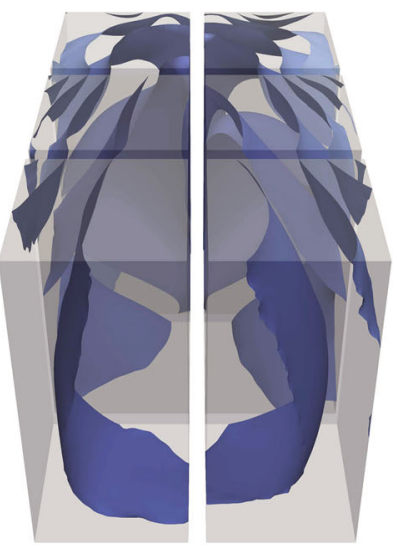

Fig. 8 Isotemperature contours every $0.1 \mathrm{~K}$ between $253.15 \mathrm{~K}$ and $255.15 \mathrm{~K}$ of the simulated loading with PUR insulation (left), with PUR+VIP insulation (mid) and with PUR+VIP insulation and LHS (right). The two rear trolleys are shown at the bottom, the two front trolley are shown at the top, respectively, and the view point is from above 

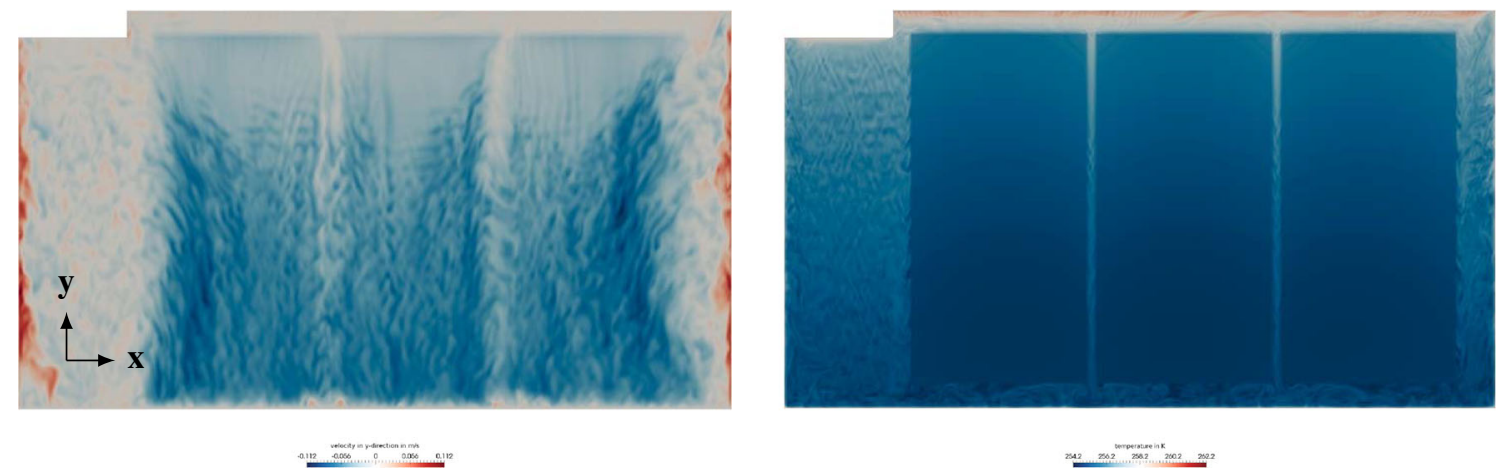

Fig. 9 Distribution of the velocity's y-component and the temperature after shutdown of the cooling unit at the mid of the truck, in the middle gab between the trolleys, slice in the $x-y$-plane

is still competitively uniform and cold as the PUR+VIP case, which is a very good result, measured by the fact that only passive cooling was provided.

\subsubsection{Influence of switching off the air conditioning}

When the AC system switches off, the refrigerated body is no longer provided with cooling capacity, but heat continues to penetrate into the volume through the walls. After a certain time, the first temperature gradients begin to appear in the inside air and in the cargo. As a starting point for these simulations, flow and temperature fields after $60 \mathrm{~s}$ of air conditioning under full load are used as initial distributions. The $\mathrm{AC}$ is then deactivated and a maximum simulation time of $11 \mathrm{~min}$ is set.

Figure 9 (left) shows a slice through the velocity field in $y$-direction at $y=\frac{l_{y}}{2}$ to demonstrate the flow in the middle channel, while Fig. 9 (right) shows the corresponding temperature field on the same slice to demonstrate temperature differences in the load after a physical time of $t=4 \mathrm{~min}$. The onset of turbulent natural convection is clearly visible. Air rises along the inner walls

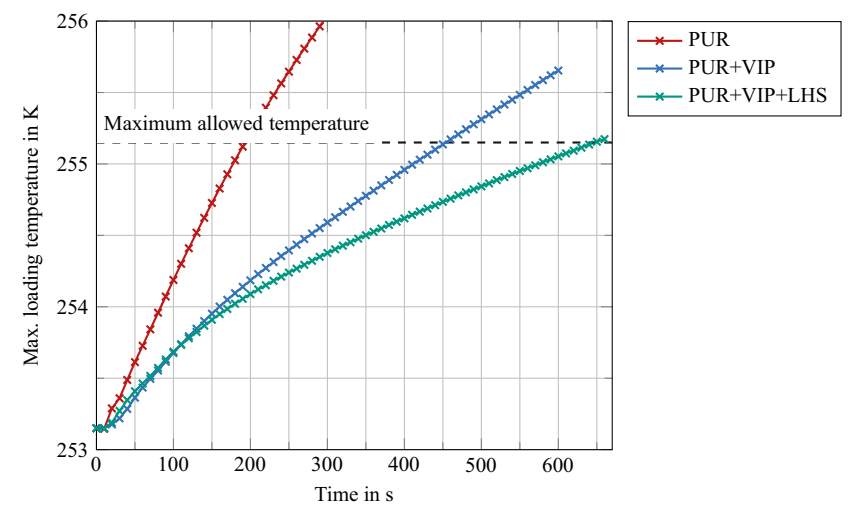

Fig. 10 Loading temperature over time for the three simulation cases PUR, PUR+VIP, PUR+VIP+LHS of the refrigerated body and reaches velocities of over $0.1 \mathrm{~m} / \mathrm{s}$, whereas the air in the gap between the cooling containers sinks by up to $0.1 \mathrm{~m} / \mathrm{s}$. The air in the gap between the cooling containers is then cooled down and sinks by up to $0.1 \mathrm{~m} / \mathrm{s}$. The temperature field of the air shows a stratification: warm air collects in the ceiling area of the refrigerated body and cooler air drops towards the floor. The Rayleigh number of the formed natural convection can be determined to $R a=3.45 \cdot 10^{9}$.

For comparison, the simulations are carried out for the setups PUR, PUR+VIP and PUR+VIP+LHS. The three configurations are compared in Fig. 10 on the basis of the maximum cooling temperature occurring over time.

It can be seen for both insulation concepts, that the increase in cargo temperature already starts after $t=10 \mathrm{~s}$, with an approximately linear increase in the maximum cargo temperature. The upper limit temperature of the cold chain is already exceeded after $t=3.33$ min with the pure rigid foam insulation. The use and sale of the warmed up frozen goods would be prohibited. In contrast, the new insulation concept with integrated VIPs reduce the incline of the cargo temperature effectively. The time after which the good's temperature exceeds the cooling limit temperature for the new insulation concept is $t=7.66 \mathrm{~min}$. A further increase in this duration can be achieved by installing the LHS on the ceiling: As shown in Fig. 10 the cooling limit temperature is exceeded after $650 \mathrm{~s} \approx 11 \mathrm{~min}$ in the LHS case.

\section{Conclusion}

In this paper, two innovative concepts for an improved refrigerated vehicle are numerically investigated - an improved insulation combined of polyurethane hard foam and VIPs and the application of a LHS in the refrigerated body. Both concepts are simulated with the double 
distribution thermal Lattice Boltzmann Method including spatially and transient conjugate heat transfer resolving the insulation walls and the deep frozen cargo.

Based on our simulations the key findings of this study are summarized as follows:

- A homogenized and highly reduced ingoing heat flux is found for the combined PUR and VIP insulation material case compared to pure PUR insulation. The present concept of VIP inclusion is found capable of halving the required cooling energy.

- The VIP inclusion is further found to enable the AC system to be replaced by a LHS mounted near the top of the refrigerator body and an additional ventilation system of lower total capacity, for which a configuration and an inclusion concept is presented in Section 4.2.

- In order to assure a homogeneous temperature in the cargo during operation, a slight flow around the refrigerated goods is found to be necessary by considering the temperature homogeneity of deep frozen food products loaded in the refrigerated body.

- In addition, the maximum downtime of the AC system is determined to about $8 \mathrm{~min}$ in case of PUR+VIP insulation and to $11 \mathrm{~min}$ in case of an additional LHS.

Thus, this study shows that the evaluated concepts are promising and that the LES-LBM approach offers a good analysis tool providing helpful insights for the design of a prototype including both concepts to reduce the fuel consumption. In cooperation with the medium-sized company Kress Fahrzeugbau GmbH as part of the research project ZF4005102CL5 funded by the Central Innovation Program for SMEs, these concepts and their practical implementation are being pursued. We look forward to presenting corresponding results in a future contribution.

Acknowledgments The authors would like to thank the German Central Innovation Programme for SMEs for funding this project (ZF4005102CL5). This work was performed on the computational resource ForHLR II funded by the Ministry of Science, Research and the Arts Baden-Württemberg and DFG ("Deutsche Forschungsgemeinschaft"). The authors declare that there is no conflict of interest.

\section{References}

1. Normblatt DIN (1995) 8959: Prüfung von wảrmegedảmmten Beförderungsmitteln für Lebensmittel. Beuth-Verlag, Berlin

2. Mohammed AA (2012) Lattice Boltzmann method: fundamentals and engineering applications with computer codes. Springer Science \& Business Media. https://doi.org/10.2514/1.J051744

3. Ahmad M, Bontemps A, Sallée H, Quenard D (2006) Experimental investigation and computer simulation of thermal behaviour of wallboards containing a phase change material. Energy Build 38(4):357-366. https://doi.org/10.1016/j.enbuild.2005.07.008
4. Al-Alawi BM, Bradley TH (2013) Review of hybrid, plug-in hybrid, and electric vehicle market modeling Studies. Renew Sustain Energy Rev 21:190-203. https://doi.org/10.1016/j.rser.2012. 12.048

5. Barad MF, Kocheemoolayil JG, Kiris CC (2017) Lattice Boltzmann and Navier-stokes cartesian cfd approaches for airframe noise predictions. In: 23rd AIAA Computational fluid dynamics conference, $\mathrm{p} 4404$

6. Bouquerel M, Duforestel T, Baillis D, Rusaouen G (2012) Heat transfer modeling in vacuum insulation panels containing nanoporous silicas - a review. Energy Build 54:320-336. https:// doi.org/10.1016/j.enbuild.2012.07.034

7. Dahmen N, Abeln J, Eberhard M, Kolb T, Leibold H, Sauer J, Stapf D, Zimmerlin B (2017) The bioliq process for producing synthetic transportation fuels. Wiley Interdiscip Rev: Energy Environ, 6(3). https://doi.org/10.1002/wene.236

8. Demirbaş A (2006) Global renewable energy resources. Energy Sources 28(8):779-792. https://doi.org/10.1080/0090831060071 8742

9. Eberhardt HF, GmbH PD (2005) Vom Pulver zur Paneele - Wie entsteht ein VIP? VIP-Bau: 2. Fachtagung - Erfahrungen aus der Praxis, pp 1-9

10. European Commision (2012) Energy Roadmap 2050. Policy (April), 1-9. https://doi.org/10.2833/10759

11. Gaedtke M, Wachter S, Rädle M, Nirschl H, Krause MJ (2018) Application of a lattice Boltzmann method combined with a Smagorinsky turbulence model to spatially resolved heat flux inside a refrigerated vehicle. Comput Math Appl 76(10):23152329. https://doi.org/10.1016/j.camwa.2018.08.018

12. Guo Z, Shi B, Zheng C (2002) A coupled lattice BGK model for the Boussinesq equations. Int J Numer Methods Fluids 39(4):325342. https://doi.org/10.1002/fld.337

13. Guo Z, Zheng C, Shi B (2002) Discrete lattice effects on the forcing term in the lattice Boltzmann method. Phys Rev E 65:46308. https://doi.org/10.1103/PhysRevE.65.046308

14. Han B, Choi JH, Dantzig JA, Bischof JC (2006) A quantitative analysis on latent heat of an aqueous binary mixture. Cryobiology 52:146-151. https://doi.org/10.1016/j.cryobiol.2005.09.007

15. Hou S, Sterling J, Chen S, Doolen GD (1994) A lattice Boltzmann subgrid model for high Reynolds number flows. arXiv:comp-gas/9401004, 1-18

16. International Energy Agency (IEA) (1998) World Energy Outlook 1998. IEA/OECD, Paris

17. James SJ, James C, Evans JA (2006) Modelling of food transportation systems - a review. Int J Refrig 29(6):947-957. https://doi.org/10.1016/j.ijrefrig.2006.03.017

18. Junk M, Yang Z (2008) Outflow boundary conditions for the lattice Boltzmann method. Progress Comput Fluid Dyn 8(11):3848. https://doi.org/10.1504/PCFD.2008.018077

19. Kalnæs SE, Jelle BP (2014) Vacuum insulation panel products: a state-of-the-art review and future research pathways. Appl Energy 116(7465):355-375. https://doi.org/10.1016/j.apenergy.2013.11. 032

20. Krüger T, Kusumaatmaja H, Kuzmin A, Shardt O, Silva G, Viggen EM (2016) The lattice Boltzmann method: principles and practice. Springer, Berlin

21. Kwon JS, Jang CH, Jung H, Song TH (2009) Effective thermal conductivity of various filling materials for vacuum insulation panels. Int J Heat Mass Transf 52(23-24):5525-5532. https://doi.org/10.1016/j.ijheatmasstransfer.2009.06.029

22. Latt J, Chopard B, Malaspinas O, Deville M, Michler A (2008) Straight velocity boundaries in the lattice Boltzmann method. Phys Rev E - Statist Nonlinear Soft Matter Phys 77(5):56703. https://doi.org/10.1103/PhysRevE.77.056703 
23. Lorenzati A, Fantucci S, Capozzoli A, Perino M (2017) The effect of temperature on thermal performance of fumed silica based vacuum insulation panels for buildings. Energy Procedia 111 (2016):490-499. https://doi.org/10.1016/j.egypro.2017.03.211

24. Ross-Jones J, Gaedtke M, Sonnick S, Rädle M, Nirschl H, Krause MJ (2019) Conjugate heat transfer through nano scale porous media to optimize vacuum insulation panels with lattice Boltzmann methods. Comput Math Appl 77(1):209-221. https://doi.org/10.1016/j.camwa.2018.09.023

25. Singh H, Geisler M, Menzel F (2015) Experimental investigations into thermal transport phenomena in vacuum insulation panels (VIPs) using fumed silica cores. Energy Build 107:76-83. https://doi.org/10.1016/j.enbuild.2015.08.004

26. Smale NJ, Moureh J, Cortella G (2006) A review of numerical models of airflow in refrigerated food applications. Int J Refrig 29(6):911-930. https://doi.org/10.1016/j.ijrefrig.2006.03.019

27. Sonnick S, Meier M, Ross-Jones J, Erlbeck L, Medina I, Nirschl H, Rädle M. (2019) Correlation of pore size distribution with thermal conductivity of precipitated silica and experimental determination of the coupling effect. Appl Therm Eng 150:10371045. https://doi.org/10.1016/j.applthermaleng.2019.01.074

28. Succi S (2001) The Lattice Boltzmann equation: for fluid dynamics and beyond. Oxford University Press. ISBN 0198503989

29. Van der Zwaan B, Keppo I, Johnsson F (2013) How to decarbonize the transport sector? Energy Polic 61:562-573. https://doi.org/10.1016/j.enpol.2013.05.118
30. VDI-Gesellschaft (2013) VDI-Wärmeatlas, 11., bearb edn. Springer, Berlin

31. Verma P, Varun Singal SK (2008) Review of mathematical modeling on latent heat thermal energy storage systems using phase-change material. Renew Sustain Energy Rev 12(4):9991031. https://doi.org/10.1016/j.rser.2006.11.002

32. Wakili KG, Bundi R, Binder B (2004) Effective thermal conductivity of vacuum insulation panels. Build Res Inf 32(4):293-299. https://doi.org/10.1080/0961321042000189644

33. Wang J, Wang M, Li Z (2007) A lattice Boltzmann algorithm for fluid-solid conjugate heat transfer. Int J Therm Sci 46(3):228234. https://doi.org/10.1016/j.ijthermalsci.2006.04.012

34. Wegger E, Jelle BP, Sveipe E, Grynning S, Gustavsen A, Baetens R, Thue JV (2011) Aging effects on thermal properties and service life of vacuum insulation panels. J Build Phys 35(2):128-167. https://doi.org/10.1177/1744259111398635

35. Zalba B, Marín J. M., Cabeza LF, Mehling H (2003) Review on thermal energy storage with phase change: materials, heat transfer analysis and applications. Appl Therm Eng 23(3):251283. https://doi.org/10.1016/S1359-4311(02)00192-8 


\section{Repository KITopen}

Dies ist ein Postprint/begutachtetes Manuskript.

Empfohlene Zitierung:

Gaedtke, M.; Wachter, S.; Kunkel, S.; Sonnick, S.; Rädle, M.; Nirschl, H.; Krause, M. J. Numerical study on the application of vacuum insulation panels and a latent heat storage for refrigerated vehicles with a large Eddy lattice Boltzmann method. 2019. Heat and mass transfer. doi: $\underline{10.5445 / / R / 1000104600}$

Zitierung der Originalveröffentlichung:

Gaedtke, M.; Wachter, S.; Kunkel, S.; Sonnick, S.; Rädle, M.; Nirschl, H.; Krause, M. J. Numerical study on the application of vacuum insulation panels and a latent heat storage for refrigerated vehicles with a large Eddy lattice Boltzmann method. 2019. Heat and mass transfer. doi: $10.1007 / \mathrm{s} 00231-019-02753-4$ 